\title{
VARIABILITY IN THE POWER SPECTRUM OF SOLAR FIVE-MINUTE OSCILLATIONS*
}

\author{
(Invited Review)
}

\author{
FRANK HILL and JURI TOOMRE
}

Department of Astrophysical, Planetary and Atmospheric Sciences**, and Joint Institute for Laboratory Astrophysicst. University of Colorado, Boulder, CO 80309, U.S.A.

and

LAURENCE J. NOVEMBER

Air Force Geophysics Laboratory and AURA, Sacramento Peak Observatory, Sunspot, NM 88349, U.S.A.

\begin{abstract}
Two-dimensional power spectra of solar five-minute oscillations display prominent ridge structures in $(k, \omega)$ space, where $k$ is the horizontal wavenumber and $\omega$ is the temporal frequency. The positions of these ridges in $k$ and $\omega$ can be used to probe temperature and velocity structures in the subphotosphere. We have been carrying out a continuing program of observations of five-minute oscillations with the diode array instrument on the vacuum tower telescope at Sacramento Peak Observatory (SPO). We have sought to establish whether power spectra taken on separate days show shifts in ridge locations; these may arise from different velocity and temperature patterns having been brought into our sampling region by solar rotation. Power spectra have been obtained for six days of observations of Doppler velocities using the $\mathrm{Mg}_{\mathrm{I}} \lambda 5173$ and $\mathrm{Fe} \mathrm{I} \lambda 5434$ spectral lines. Each data set covers 8 to $11 \mathrm{hr}$ in time and samples a region $256^{\prime \prime} \times 1024^{\prime \prime}$ in spatial extent, with a spatial resolution of $2^{\prime \prime}$ and temporal sampling of $65 \mathrm{~s}$. We have detected shifts in ridge locations between certain data sets which are statistically significant. The character of these displacements when analyzed in terms of eastward and westward propagating waves implies that changes have occurred in both temperature and horizontal velocity fields underlying our observing window. We estimate the magnitude of the velocity changes to be on the order of $100 \mathrm{~m} \mathrm{~s}^{-1}$; we may be detecting the effects of large-scale convection akin to giant cells.
\end{abstract}

\section{Introduction}

Ridge structures are evident in spatial and temporal $(k, \omega)$ power spectra of the observed five-minute oscillations of the Sun (e.g., Deubner et al., 1979). Such ridges in power arise because acoustic waves trapped in the thermal structure below the solar surface possess a fairly specific relationship between their frequency $\omega$ and horizontal wavenumber $k$. Since the character of the oscillations is determined largely by the vertical stratification of temperature below the solar surface, the observed positions of the ridges could be compared with theory to determine at least the adiabat in the isentropic part of the convection zone (e.g., Berthomieu et al., 1980; Lubow et al., 1980). However, the dispersion relations linking $k$ and $\omega$ will be perturbed by temperature and velocity structures associated with the convection, and so too by differential rotation. The purpose of this observational study is to attempt to detect shifts in ridge positions that

* Proceedings of the 66th IAU Colloquium: Problems in Solar and Stellar Oscillations, held at the Crimean Astrophysical Observatory, U.S.S.R., 1-5 September, 1981.

** Formerly Department of Astro-Geophysics.

$\dagger$ JILA is operated jointly by the University of Colorado and the National Bureau of Standards. 
may be attributed to large-scale convective fluctuations of temperature and velocity. A preliminary theoretical assessment of the relative ridge displacements that may arise from such convection is presented in an accompanying paper by Gough and Toomre (1983).

The apparent frequency $\omega$ of an acoustic mode trapped below the surface can be modified in several ways. The simplest is that of a Galilean translation of the wave fronts: a uniform horizontal flow of amplitude $U$ will advect the wave fronts, producing a change in $\omega$ with respect to a stationary observer. This change $\Delta \omega$ scales as $k U$, but its sign depends upon the direction of propagation of the wave relative to $U$. If this flow varies with depth $z$, the effective velocity that is sampled by each mode may be different for it depends on an average of $U(z)$ weighted with the kinetic energy density of the wave with depth (cf. Gough, 1978). In contrast, steady flows with a cellular pattern in the horizontal produce local dilations and contractions in the wavefronts, thereby modulating the local $\omega$. However, if the horizontal scale of such a cellular flow is much larger than that of the trapped mode being sampled, then $(k, \omega)$ power spectra formed over different portions of the Sun should display reasonably simple ridge shifts in $\omega$. Thus giant cells with proposed scales of $300 \mathrm{Mm}$ or greater may be capable of being detected by using modes with horizontal wavelengths for instance between 5 to $25 \mathrm{Mm}$. On the other hand, supergranular flows with typical $50 \mathrm{Mm}$ scales would primarily broaden such ridges in $\omega$, owing to the large horizontal areas that must be sampled by the observations before the $k$ resolution of the Fourier transforms becomes small enough to discern ridges. Finally, the presence of horizontal temperature variations across a cell also produces dilations and contractions in the wavefronts of the acoustic modes, most simply because of changes in the local sound speed. Once again the local change in $\omega$ is determined by an integral with depth of the perturbation in sound speed weighted by the kinetic energy density of the wave, but the sign of $\Delta \omega$ is independent of the propagation direction. Thus the oscillations could also serve as probes of temperature structures, though here too interpretations are simplest if the horizontal scale of the inhomogeneity is much larger than that of the modes.

Since the variations produced in $\omega$ by horizontal flows depend upon the wave propagation direction while those for temperature do not, it should be possible to separate the two effects of convection by measuring local dispersion relations for waves travelling in opposite directions. Theoretical details concerning the perturbations to the dispersion relations are given in the accompanying paper by Gough and Toomre (1983), along with illustrations of the kernels that enter into the integrals that determine $\Delta \omega$. An outline of the inversion procedure required to deduce the velocity and temperature variations with depth from observed ridge shifts is discussed in Gough (1978).

In this observational search for effects of large-scale convection on the spectrum of five-minute oscillations, we will restrict ourselves to modes of high degree. The oscillations are then trapped in a relatively shallow resonant cavity, or wave guide, just beneath the photosphere. The lower reflection occurs at a depth of about $n R / l$, where $n$ and $l$ are the order and degree of the mode and $R$ is the radius of the Sun. The horizontal wavenumber of such modes is $k \simeq l / R$. The observations will be used to 
determine $(k, \omega)$ power diagrams for individual days, and the ridge structures in these will be compared from day to day to look for any significant displacements. We will concentrate on modes with horizontal wavenumbers ranging from about 0.25 to $1.25 \mathrm{Mm}^{-1}$, with the corresponding $l$ values ranging from about 175 to 875 , and for $n$ values of 1 to 4 .

\section{Observations and Reduction}

Our observations are made with the SPO diode-array, which operates at the exit of the echelle spectrograph attached to the vacuum tower telescope. Four strings of 128 diodes each are arranged parallel to the spectrograph slit. One pair of diode strings, with $0.052 \AA$ masks, is located at $\pm 0.079 \AA$ in the magnetically insensitive Fe I $\lambda 5434.5$ line, which allows us to measure Doppler velocities in the lower photosphere (Altrock et al., 1975). The second pair of diode strings, with $0.120 \AA$ masks, is located at $\pm 0.060 \AA$ in the $\mathrm{Mg}$ I 25172.7 line, which is representative of the temperature minimum region (Altrock and Canfield, 1974). Two-dimensional intensity and velocity images are obtained by spatially scanning the solar image across the spectrograph slit. The scanning direction is parallel to the solar equator and covers 512 steps, producing nominally a $256^{\prime \prime} \times 1024^{\prime \prime}$ image with a spatial resolution of $2 "$. Such an observing window elongated in the east-west direction will permit detection of waves with a wide range of horizontal wavenumbers $k$ which are propagating parallel to the equator; averaging of the data in the north-south direction will serve to largely filter out waves traveling in oblique directions. These scans are repeated every $65 \mathrm{~s}$ for periods as long as possible given the constraints of weather and the extent of the daylight hours; the observational sets typically span 8 to $11 \mathrm{hr}$. The scanning region remains centered on the disc throughout the observing day.

The determination of instantaneous Doppler velocities and intensities at each spatial position requires forming appropriate sums, differences and ratios of the diode measurements carried out on each side of the spectral line. Details of the procedures used are discussed in November et al. $(1979,1982)$, together with an analysis of the major instrumental errors affecting the velocity measurements. We find that sensitivity variations in the diodes and random seeing effects in the spectrograph are the dominant sources of instrumental error at spatial scales (in the scan direction) smaller than about $200 \mathrm{Mm}$, or spatial wavenumbers $k \gtrsim 3 \times 10^{-2} \mathrm{Mm}^{-1}$; we estimate that the signal-tonoise ratio in our determination of instantaneous Doppler velocities is about 30 for such spatial scales. Noise is also introduced by spectrograph grating jitter, the latter driven by mechanical flexure of the spectrograph tube. As a consequence of our scanning procedure to build up to two-dimensional velocity image, such flexure at low temporal frequencies results in noise which may dominate the velocity data at spatial scales greater that about $200 \mathrm{Mm}$. Since the flexure is a stochastic process, noise is distributed over a broad range of temporal frequencies. Incremental stepping of the observing table to compensate for rotation of the solar image also excites some grating jitter. Because the stepping is carried out at precise intervals of $1 \mathrm{~s}$ in time and is synchronized with 
the start of each spatial scan, this noise appears as power at zero temporal frequency and at selected spatial frequencies.

The apparent position of a ridge in the $(k, \omega)$ domain will be affected by our ability to define accurately the temporal and spatial scales in our observations. We believe that our time scale is very stable, for the telescope control computer sequencing has access to a precision clock and we monitor the repetition timing for the scanning with care. The stability of the spatial reference scale is a more intricate issue. Basically this scale is set by the apparent diameter of the Sun. Changes in the magnification of the telescope or the spectrograph, variations in atmospheric refraction, and changes in the travel of the scanning platform for the spectrograph entrance slit can each modify the apparent spatial scale. Given the thermal and optical design of the vacuum telescope and the echelle spectrograph, we estimate that thermal expansion effects in the structures produce variations in the apparent solar diameter of less than $0.05 \%$. Reproducibility of magnification in the diode-array apparatus also does not constitute a problem, since thermal stresses are negligible and all equipment has been undisturbed for the entire time span of the observations. Differential atmospheric refractive effects will change the image scale during the course of the observations in a fairly predictable manner (Simon, 1966; Saastamoinen, 1979), but this effect is only significant very close to the horizon. We have corrected for the shape of the Sun given its position in the sky, and estimate that residual errors in image scale due to uncorrected atmospheric refraction effects in our observations are less than $0.03 \%$. In addition, we apply daily corrections for changes in the apparent radius of the Sun due to the Earth's orbital position. Further, the vacuum telescope is now equipped with a limbtracking guiding telescope that is locked to the main optical beam by an actively monitored laser system, and we find that the main telescope pointing is better than the seeing limit throughout the course of a day's observations. The precision with which we can return to the same starting point for the scans and the extent of travel of the scanning platform are now reproducible to the extent that the spatial reference scale may be modified at most by $0.02 \%$.

The construction of $(k, \omega)$ power diagrams from our data proceeds in the following manner. The two-dimensional velocity raster formed every $65 \mathrm{~s}$ is averaged in the direction of the shorter dimension perpendicular to the equator. This has the effect of averaging out the signature of most waves propagating obliquely to the slit and preserves those waves traveling perpendicular to it, or parallel to the solar equator. We form a two-dimensional array with these collapsed rasters, with each row of the array containing the averaged data from one scan in time. We extend our data set with zeroes if necessary to a length of 644 points so that the resolution bin width in $\omega$ is constant for all runs to permit detailed comparison of results from different days. Thus the resolution bin width in $\omega$ is $1.5 \times 10^{-4} \mathrm{~s}^{-1}$.

The length of our scans parallel to the equator is about one solar radius. We therefore have to correct our data for foreshortening effects, and do so by interpolating our data from its observed rectangular grid onto a projected spherical grid with equal longitude bins of width 0.133 degrees. The width of the corresponding horizontal wavenumber $k$ bins is $7.6 \times 10^{-3} \mathrm{Mm}^{-1}$. The data are apodized or tapered to zero at the edges of the 
collapsed array by using either a cosine bell window or a variety of exponential functions extending typically $10 \%$ into the data. The apodization is also applied around any gaps that may be present due to clouds or other causes. The ridge locations are insensitive to the various apodizing procedures that we have examined, although power between the ridges is influenced by the functional form. After extending, interpolating and apodizing the data, we apply a two-dimensional fast Fourier transform to it and produce power and phase pictures from the complex transform.

We studied differences of ridge locations in $(k, \omega)$ power diagrams from two different days by at first blink comparing them on a color imaging system. This procedure suggested that there were relative changes in ridge positions, but these differed from ridge to ridge and quadrant to quadrant. However, this qualitative impression is easily influenced by changes in the width of the ridges and by the choice of scaling for the displays. Consequently, we developed the following quantitative procedure for determining differences in the position of a given ridge on two different days. First, a contour map of a $(k, \omega)$ diagram is produced on a graphics device. The cursor of the device is used to draw a polygonal box enclosing the ridge of interest, and the positions of the vertices of the box in the $(k, \omega)$ diagram are recorded. The region of the diagram enclosed in the box is extracted from the full diagram, and the power as a function of $\omega$ for each fixed $k$ in this region is then examined. An integral moment method is used to define the centroid of power in a given ridge at each value of $k$ : we adopted the ratio of the first moment to the zeroth moment to specify the centroid position. These points are then fitted with a least squares variable knot cubic spline. This fitting algorithm applies a least squares fit to the segments of data between knot locations. The cubic spline conditions are satisfied at the knots, and the positions of the knots are varied from some initial distribution until the least squares error is minimized. This error is the root mean square value of the deviation between the local ridge centroids and the calculated fit. We typically use 5, 10, and 28 knots in our cubic spline fits for data sets involving about 130 points on a given ridge

\section{Results}

Our data base currently consists of six days of observations carried out in February and March 1981. Details of these data sets are provided in Table I which lists the dates and

TABLE I

Summary of oscillation data sets

\begin{tabular}{lcl}
\hline Date & Time extent & Time samples \\
\hline * 22 Feb. 81 (Day 53) & $9.4^{\mathrm{h}}$ & 522 \\
23 Feb. 81 (Day 54) & $6.9^{\mathrm{h}}$ & 384 \\
* 24 Feb. 81 (Day 55) & $9.9^{\mathrm{h}}$ & 547 \\
* 23 Mar. 81 (Day 82) & $11.4^{\mathrm{h}}$ & 632 \\
25 Mar. 81 (Day 84) & $10.0^{\mathrm{h}}$ & 512 \\
26 Mar.81 (Day 85) & $10.7^{\mathrm{h}}$ & 550 \\
\hline
\end{tabular}


corresponding day numbers in the year, the total extent in time of the observations, and the number of spatial raster scans (or time samples) available in each set. We have chosen to present here comparisons involving the three data sets marked with asterisks in Table I. These sets are the longest in time extent and show the least noise. Comparisons of ridge locations involving the other days yield results which are less certain because of increased observational noise, but these generally support the conclusions based on Days 53,55 , and 82 . We presently have a further seven data sets that need to be analyzed from more recent observations in this continuing program.

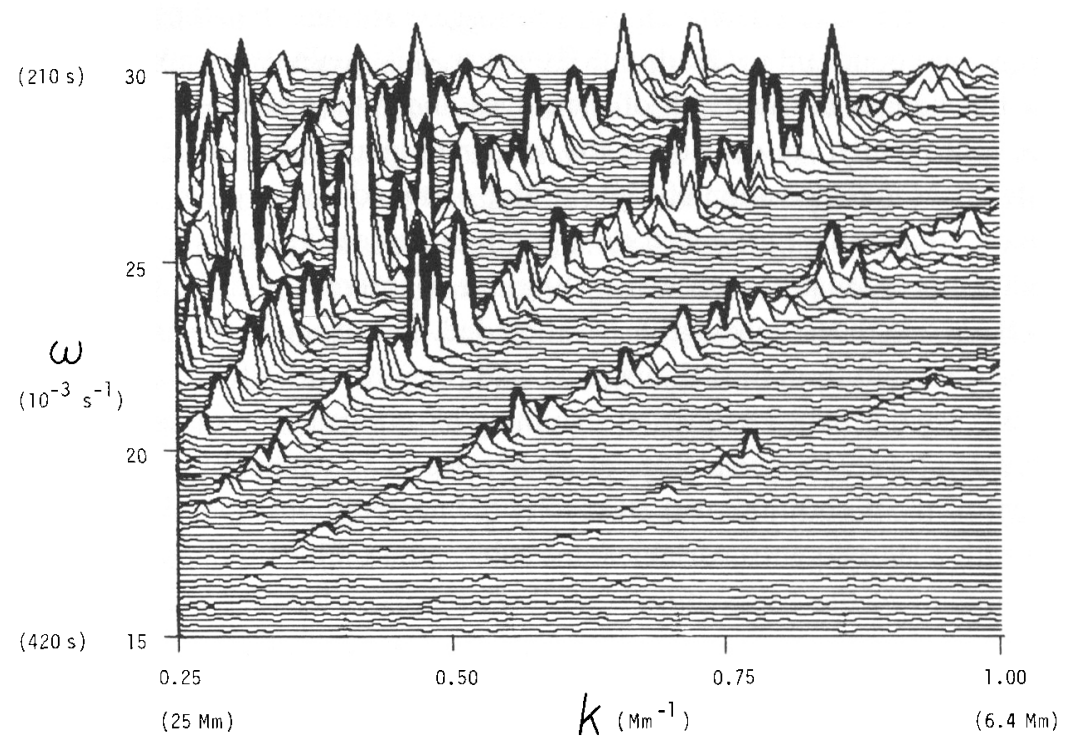

Fig. 1. Portion of positive $\omega$ quadrant of $(k, \omega)$ power diagram for high degree solar oscillations observed in MgI Doppler velocities on Day 82 (23 March, 1981). The $p_{0}$ to $p_{4}$ ridges appear distinctly in the foreground, while other ridges are partially obscured in this perspective view. The power levels have been multiplied by the arbitrary factor $k$ to enhance the ridges at high values of $k$. An uneven distribution of power is evident along the ridges. These variations are probably due to the effects of interference from mode beating within the resolution bins and bin coupling from seeing.

Figure 1 displays a portion of one of the quadrants of the $(k, \omega)$ power diagram for Doppler velocities observed in $\mathrm{Mg}$ I on Day 82. The section shown is an area with a high signal-to-noise ratio where instrumental error effects are negligible. We can easily discern four ridges in this perspective view, with another three ridges partially hidden in the background of the plot. A full contour plot of this quadrant clearly shows 11 ridges. The fundamental, or $p_{0}$, ridge possesses substantially less power than the other ridges, in keeping with previous observational results (e.g. Deubner et al., 1979; Rhodes et al., 1981). There is also considerable variation in power along any given ridge, with the power often appearing to be distributed in a succession of peaks and troughs. Such variations in power are partly due to beating between discrete modes whose 
separations in $k$ and $\omega$ are too small to be resolved by our observations. The fact that we can only observe a finite region of the Sun for a finite length of time results in resolution elements (or bins) in $k$ of $2 \pi / X$ and in $\omega$ of $2 \pi / T$, where $X$ is the length of our observing raster and $T$ is the time interval covered by one day of observations. Thus our sampling bins are larger than the separation in $(k, \omega)$ space of individual modes, each bin containing on the order of 30 modes, given the aspect ratio of our observing window and its efficiency in filtering out waves propagating obliquely to the equator. The presence of many modes with small separations in $k$ and $\omega$ in each bin results in a complicated beating pattern in both space and time. In principle, mode beating by itself leads to net power that would probably show little correlation from bin to bin. Thus we would expect the power in a given bin to be different on different days, possibly even occasionally vanishing.

In addition to mode beating, seeing effects will distribute power unevenly along the ridges. Figure 1 shows regions along the ridges where power is concentrated into structures with scales of about 5 to 10 bins in $k$. It thus appears that power in adjacent bins along a ridge are not independently determined, in the manner that we might expect from mode beating effects. There is coupling or leakage between the bins on a scale that cannot be just attributed to our discrete sampling in $k$ and $\omega$, which may result in the coupling of about 3 bins together, rather than the 5 to 10 that we observe. The observed structures in power along the ridge are likely to be the results of seeing effects in the Earth's atmosphere. There are three stochastic effects of seeing that can couple bins in the observed $(k, \omega)$ power diagram. The first effect is that of blurring, which involves small relative displacements of the image and consequent blending of image elements. Such blurring removes power from small wavelengths or high $k$ values; it also changes the observed phase of the wave, and thus smears the power in both $k$ and $\omega$. Further, blurring also alters abruptly the sensitivity of our velocity measurements, for differential image motion typically broadens the spectral line sampled by the diode detectors. This too serves to change the apparent phase in the velocity signal discontinuously, thereby coupling nearby bins in both $k$ and $\omega$. Finally, the most important effect is that of sudden displacements of larger portions of the solar image: lateral image displacements of 5 " or more are common, with the image motion being correlated on horizontal scales typically of about 100" (e.g., Brandt, 1969; Tarbell and Smithson, 1981; Tarbell, 1981). Displacements of such large areas of the image will effectively transfer power from a bin to many of its neighbors. These effects of seeing contribute to the observed structure of power along the ridges, and must be recognized when trying to determine the centroid of a ridge.

Shifts in the locations of ridge centroids between Days 53 and 55 are shown in Figures 2 and 3. Cubic spline fitting procedures with 5 knots have been used to determine the ridge shifts displayed here, with the centroid positions at each $k$ having been calculated using the integral moment method discussed earlier. Figure 2 presents frequency shifts for the $p_{1}, p_{2}, p_{3}$, and $p_{4}$ ridges in the positive $\omega$ quadrant, while Figure 3 shows the shifts for the corresponding ridges in the negative $\omega$ quadrant. The solid curves show shifts observed in MgI velocity, the dotted curves are the shifts 

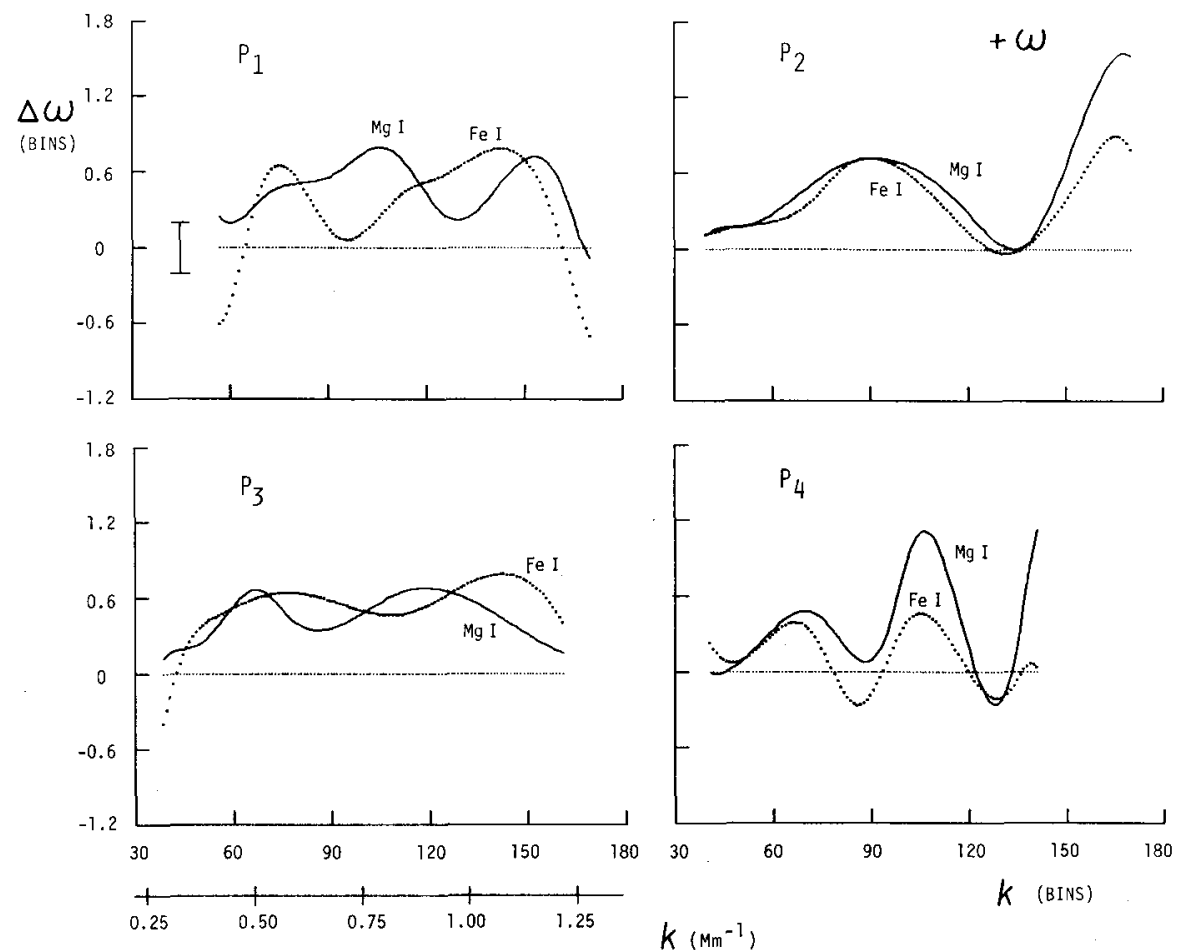

Fig. 2. Shifts in the location of the $p_{1}$ to $p_{4}$ ridges in the positive $\omega$ quadrant between Days 53 and 55 (22 February, 1981 and 24 February, 1981). These shifts result from changes in velocity and temperature perturbations experienced by waves propagating in the westward direction. The solid line is the shift observed in $\mathrm{Mg}$ I Doppler velocity data, the dotted line is the shift simultaneously observed in Fe I Doppler velocity. The abscissa is scaled by the value of $k$ in spatial bin numbers, with one bin equal to $7.6 \times 10^{-3} \mathrm{Mm}^{-1}$. In addition, a corresponding horizontal wavelength scale is superposed below. The ordinate display shifts in $\omega$, or $\Delta \omega$, in terms of frequency resolution bins, with one bin corresponding to $1.5 \times 10^{-4} \mathrm{~s}^{-1}$. The thin dotted line demarks the reference level of a null shift. The overall observational errors are denoted by the vertical bars. The character of the shifts with $k$ are different for the various ridges shown here, thus suggesting that the shifts are solar rather than instrumental in origin. The Fe I and $\mathrm{MgI}$ data display similar trends in their ridge shifts.

reduced from simultaneous observations of FeI velocity. The ordinate display shifts in $\omega$ in terms of frequency resolution bins, with one bin corresponding to $1.5 \times 10^{-4} \mathrm{~s}^{-1}$; the abscissa is scaled by the value of $k$ measured in spatial bin numbers, with one bin corresponding to $7.6 \times 10^{-3} \mathrm{Mm}^{-1}$. In addition, a horizontal wavelength scale is superposed on the abscissa.

When observed in the $\mathrm{Mg} I$ and FeI spectral lines, the shifts for a given ridge in Figures 2 and 3 display the same general trends, with the possible exception of the $p_{1}$ ridge in the positive $\omega$ quadrant. This is comforting since the two spectral lines are formed at heights in the solar atmosphere differing by only about $400 \mathrm{~km}$, and at both heights these acoustic wave modes are evanescent. Figures 2 and 3 indicate that the displacement of the different ridges is distinctive in character. The $p_{2}$ ridge shifts in both 

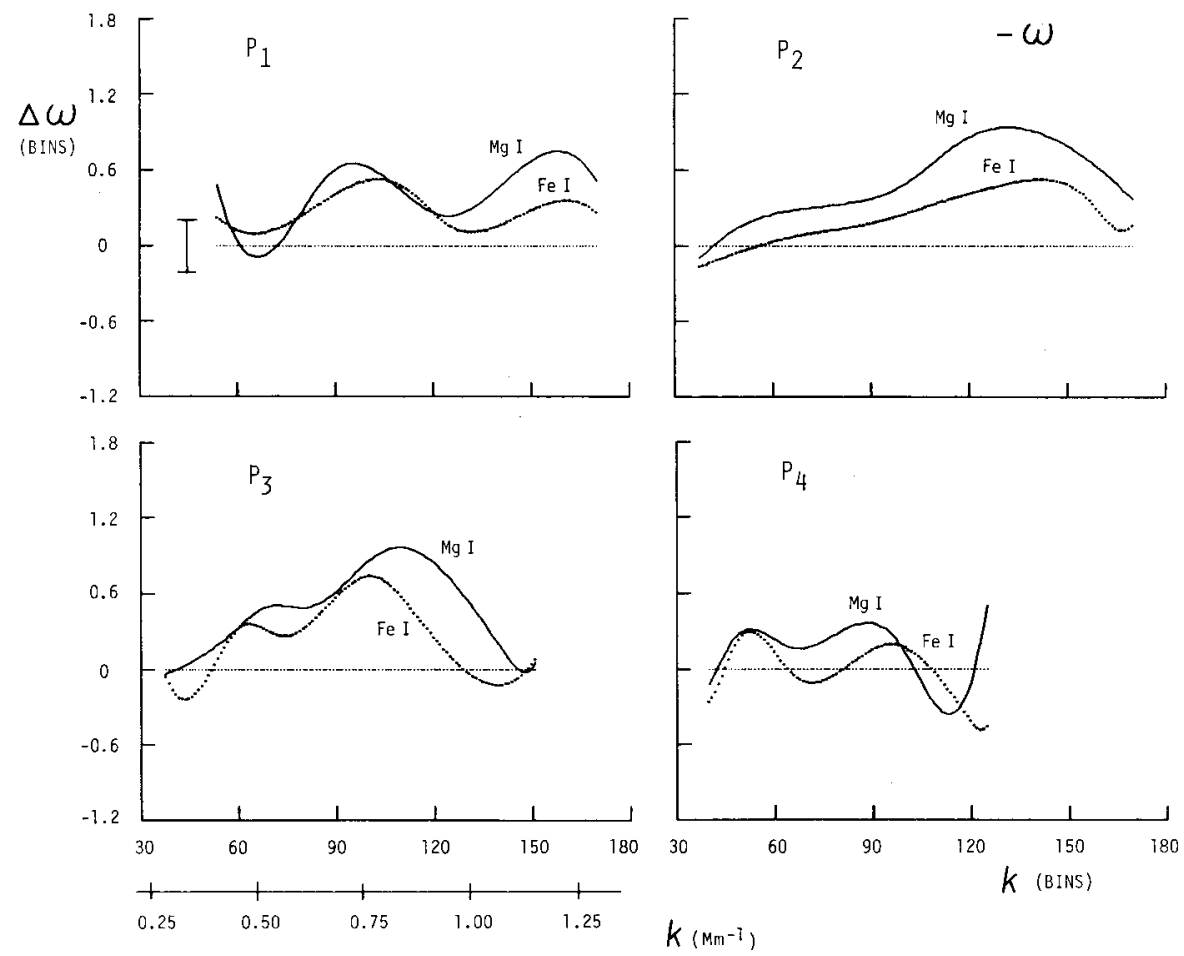

Fig. 3. Similar to Figure 2, but for ridge shifts observed in the negative $\omega$ quadrant corresponding to waves propagating in the eastward direction. Comparison of these shifts with those in Figure 2 reveal differences that are attributable to the combined effects of velocity and temperature perturbations.

quadrants show a small shift at low values of $k$ and a large shift at high $k$. The $p_{4}$ ridge in the positive $\omega$ quadrant and the $p_{3}$ ridge in the negative $\omega$ quadrant have their maximum shifts near the middle of the ridge. The other ridges shifts display a number of bends along their length. The fact that the ridge shifts have different behavior suggests that the apparent shifts do not arise from instrumental effects which would tend to shift all ridges in a similar manner.

Our estimates of confidence level in the observed ridge displacements is indicated by the error bars shown in Figures 2 and 3. Shifts with magnitudes below that level are probably not significant. Estimating our confidence level in these shifts is somewhat intricate. One possible choice of such error estimates is to calculate the rms difference between the individual centroid determinations and the smoothed spline fits to these points, thereby defining a scatter about the fit. The error, $\sigma_{1}$, estimated from such scatter, stems from all the processes that control the signal-to-noise ratio in our velocity measurements, supplemented by effects of mode beating and bin coupling. We further must add to that our estimate of errors, $\sigma_{2}$, arising from the uncorrected instrumental changes of scale. An observed shift in ridge position would then be significant if the magnitude of the shift exceeds the combined error. However, the variations in power due to mode beating and the coupling of bins from effects of seeing suggest that a simple 
rms scatter between each centroid point and its corresponding fitted point will overestimate $\sigma_{1}$, for the centroid points are not statistically independent. Thus we believe that it is appropriate to locally smooth the centroid data, using a smoothing comparable to twice the bin coupling coherence length. We then apply one of the cubic spline fitting functions, recognizing that such functions are particularly responsive to the large-scale trends along the ridges, with these trends more likely to arise from solar effects. The estimates of $\sigma_{1}$ are then based on the rms difference between the cubic spline fits and the smoothed data points. Thus the total rms error in the ridge shifts is approximately \pm 0.2 bins in $\omega$ compared to \pm 0.7 bins when calculated on the basis of individual centroid points with no smoothing. The general trends with $k$ for the ridge shifts are not overly sensitive to the number of knots used in the spline fits, nor to their initial distribution, provided we have reduced some of the effects of bin coupling.

The shifts in ridge location reflect physical structures below the solar surface. As shown in Gough and Toomre (1983), the proportional shifts $\Delta \omega / \omega$ in local wave properties can be related to subsurface velocity and temperature fields with spatial scales
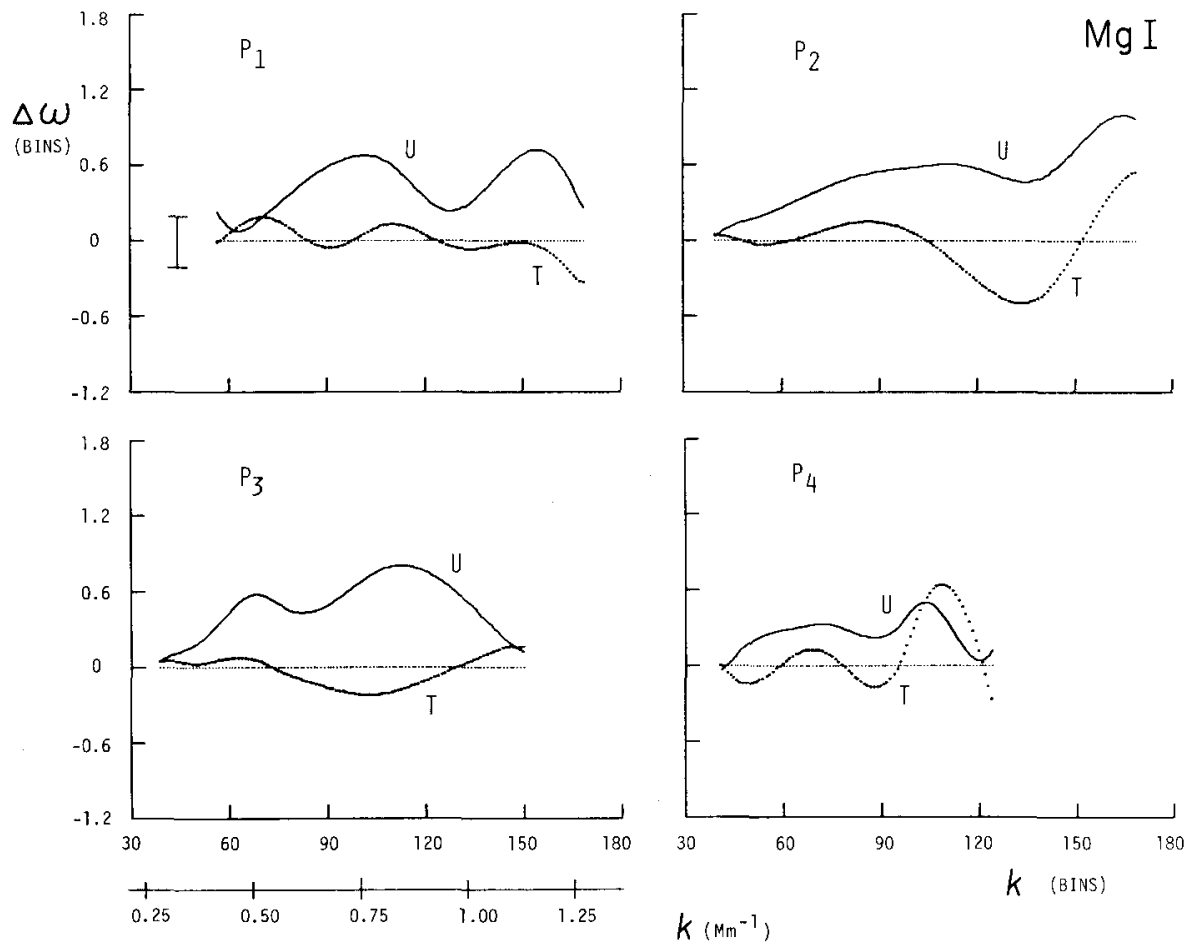

Fig. 4. Separation of ridge shifts with $k$ into the displacements attributable to velocity and temperature perturbations. The combined shifts were observed in Mg I Doppler velocity and displayed in Figures 2 and 3. The solid line (labelled $U$ ) is the shift due to velocity, the dotted line $(T)$ is that due to temperature. All scales are as in Figure 2. The velocity perturbation effects are generally larger than those due to temperature. The displacements related to velocity differences can be associated with a depth averaged velocity $\tilde{U}$ weighted by the kinetic energy density of each wave mode. Table II provides some interpretation of such velocity differences. 
much larger than the wavelengths of the observed modes. The inhomogeneities arising from convection are linked to the ridge shifts by a set of integrals possessing kernels that are fairly complicated functions of depth, and quite unlike delta functions. The proportional frequency shifts arising from advection of wave fronts by horizontal flows depend in sign upon the wave propagation direction, whereas the shifts due to temperature modifying the local sound speed do not. Thus we can separate the velocity and temperature contributions to the ridge shifts by adding and subtracting the shifts for the positive $\omega$ and negative $\omega$ ridges at a given value of $k$. The results of this separation are shown in Figures 4 and 5 . These figures present the velocity perturbation of $\Delta \omega$ as a solid line and the temperature perturbation as a dotted line. Figure 4 displays these perturbations for the $\mathrm{Mg}$ I data, and Figure 5 shows the corresponding curves for those of $\mathrm{Fe}$; again we are here comparing Days 53 and 55. The general trends with $k$ are quite similar for $\mathrm{MgI}$ and FeI. The frequency shifts due to changes in velocity are typically greater than those for temperature. These convective velocity and temperature perturbations are superimposed on top of differential rotation effects, and hence cannot be isolated without comparing two or more days of observations. It is in this regard that the estimates of differential rotation reported by Deubner et al. (1979) may be brought somewhat into question, for they used data from individual days without seeking to isolate effects that may arise from convection.
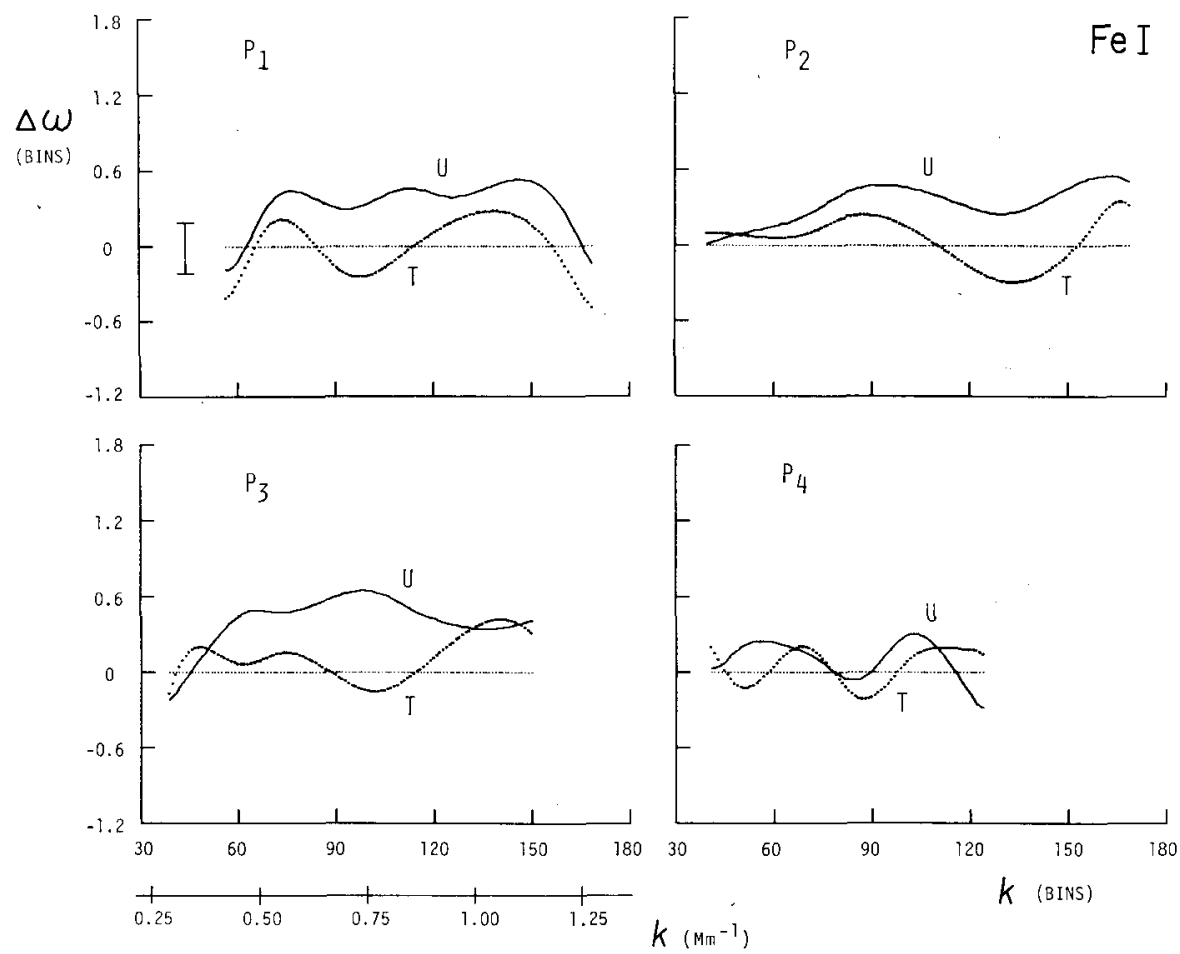

Fig. 5. Similar to Figure 4, but the separation of ridge shifts into velocity $(U)$ and temperature $(T)$ effects has been carried out for the FeI data. 
We recognize that deducing specific temperature and velocity estimates as functions of depth from the observed ridge shifts requires careful application of inverse theory. Although we have not yet carried out such inversion procedures, we can at least provide some estimates of the velocities that may produce the observed shifts. Namely, for a mode with wavenumber $k$, the frequency shift $\Delta \omega$ arising from simple advection of wave fronts by a horizontal velocity $U(z)$ is just $\Delta \omega=k \tilde{U}$, where $\tilde{U}$ is the average of $U$ weighted with the kinetic energy density of the wave. As shown in Gough and Toomre (1983), a given mode clearly samples a range of depths, and thus its associated $\tilde{U}$ does not correspond to a single velocity at a specific depth. However, the high degree modes that we consider possess lower reflection points at depths of about $n / k$, and thus the depths sampled range from about $1 \mathrm{Mm}$ to $16 \mathrm{Mm}$. We present in Table II the differences in the average velocities $\tilde{U}$ inferred from the observed shifts between Days 53 and 55 in the positions of the 4 ridges at selected values of $k$ for the $\mathrm{Mg}$ I velocity data. The absence of entries in the table represents locations where ridge centroids could not be reliably determined. Errors in determining $\tilde{U}$ are directly related to the rms error in locating the ridge centroids; we estimate that such errors in the $\widetilde{U}$ entries in Table II increase linearly with $k$, from a value of $\pm 15 \mathrm{~m} \mathrm{~s}^{-1}$ at $k=0.3 \mathrm{Mm}^{-1}$ to $\pm 50 \mathrm{~m} \mathrm{~s}^{-1}$ at $k=1.2 \mathrm{Mm}^{-1}$.

We note that all the differences in velocity $\tilde{U}$ in Table II are of the same sign. Our observational setup and subsequent use of the Fourier transform assigns the positive $\omega$ quadrant to those wave modes propagating in the westward direction. This also means that a positive velocity difference in Table II indicates an increase in the westward velocity being experienced by a mode between the two days being compared. Since we are sampling somewhat different regions on the Sun on the two days, we must emphasize that such differences in velocity probably do not represent temporal changes, but rather arise from the sampling of different convective cells. Table II reveals differences in various depth averaged horizontal velocities that range from $30 \mathrm{~m} \mathrm{~s}^{-1}$ to $170 \mathrm{~m} \mathrm{~s}^{-1}$ for the ridge intervals that were analyzed.

TABLE II

Differences in averaged horizontal velocities $\widetilde{U}$ (in $\mathrm{m} \mathrm{s}^{-1}$ ) inferred from ridge shifts in $\mathrm{Mg} \mathrm{I}$

\begin{tabular}{lrrrr}
\hline$k\left(\mathrm{Mm}^{-1)}\right.$ & \multicolumn{1}{c}{$p_{1}$} & \multicolumn{1}{c}{$p_{2}$} & \multicolumn{1}{c}{$p_{3}$} & $p_{4}$ \\
\hline 0.3 & - & - & 30 & - \\
0.4 & - & 70 & 80 & 85 \\
0.5 & 30 & 100 & 170 & 90 \\
0.6 & 90 & 120 & 110 & 70 \\
0.7 & 130 & 120 & 110 & 50 \\
0.8 & 125 & 110 & 140 & 95 \\
0.9 & 70 & 100 & 130 & - \\
1.0 & 40 & 70 & 80 & - \\
1.1 & 80 & 80 & 30 & - \\
1.2 & 90 & 115 & - & - \\
\hline
\end{tabular}


Comparison of the ridge positions between Days 53 and 82 again show significant shifts, while those between Days 55 and 82 do not. This suggests that there may be something distinctive about the region of the Sun spanned by our observing window on Day 53. Having examined synoptic magnetic maps of the solar surface for the months of February and March 1981, we find that the observing area for Day 53 spans a region on the quiet Sun showing fairly rapid evolution of the large-scale magnetic field pattern. It has been suggested that giant cells may be revealed by the evolution of magnetic structures, though more direct measurements of such cells have been inconclusive (e.g., Bumba and Howard, 1969; Bumba, 1970; McIntosh, 1980). We believe it is possible that a structure akin to a giant cell was fortuitously placed beneath our observing area on Day 53, and was then moved to an unobservable location by solar rotation on Days 55 and 82. In support of this conjecture, we note that our method of probing responds best to subphotospheric velocity and temperature structures which have a spatial scale that is a significant fraction of the $\sim 700 \mathrm{Mm}$ length of our observing window. There are ample theoretical reasons for expecting large-scale convection cells to be present that would possess such dimensions (e.g., Gilman, 1980; Toomre, 1980; Latour et al. 1981, 1983), and a number of such cells should be active at any given time. However, our method of probing these cells by observing local changes in wave properties has a sensitivity that varies with the orientation and scale of these features within the observing window. This may lead to only occasional days showing distinct shifts in $(k, \omega)$ ridges, and thus an extended program of observations is required to establish the statistics of such events.

\section{Conclusions}

Our observations have revealed that significant changes in the positions of ridges for five-minute oscillations of high degree can be detected by comparing two or more data sets. The only previous study to have compared $(k, \omega)$ diagrams from two different days reported no changes in ridge locations (Rhodes et al., 1981). However, the method used there was one of simple subtraction of one power diagram from the other, rather than seeking to fit the ridge positions in some quantitative manner. Such a subtraction method suffers from the effects of mode beating and from fluctuations in overall power levels caused by seeing variations, differences in data set lengths and varied instrumental effects. In addition, the shifts we have reported here are small and can be easily masked by the choice of scaling used to examine the differences. Any one of these factors could serve to hide a small displacement in ridge positions, and thus it is not surprising that Rhodes et al. (1981) did not detect shifts.

We believe that our detection of ridge shifts on one day out of three suggests that such observational procedures to probe for subphotospheric velocity and temperature structures holds out considerable promise. We recognize that major improvements are required in the instrumentation to reduce the noise component in the observed power diagrams, for the successful implementation of inverse theory is likely to hinge on that factor. Our suggestion that the observed ridge shifts may be related to the elusive giant 
cells is speculative at best, though it is clear that only velocity and temperature features with a horizontal scale that is a substantial fraction of our observing window could produce such ridge shifts. We think it also reasonable that only the occasional data set may show discernible shifts in ridges, for our ability to detect shifts is influenced by the orientation and scale of the convective structures. For instance, the presence of several flow patterns within our observing window may lead to dilation and contraction of wave fronts that appear to just broaden the ridges, with little perceptible shift in ridge centroids. Clearly the data base of such observations must be considerably extended, and we are so engaged.

\section{Acknowledgements}

We thank Dr Douglas Gough for extensive scientific advice and discussions concerning the interpretation of these observations, and Drs Dick Dunn, Jack Harvey, and George Simon for suggestions about potential sources of error inherent in carrying out such observations. Patrick McIntosh kindly helped us to interpret synoptic maps of magnetic field evolution. Richard Mann, Horst Mauter, and Fritz Stauffer contributed vital observing skills and patience in calibrating and stabilizing the diode array at SPO. F.H. and J.T. were Guest Observers during the course of the observations at SPO, the latter being operated by the Association of Universities for Research in Astronomy, Inc., (AURA), under contract with the National Science Foundation. L.J.N. was supported as a NAS/NRC Research Associate at Air Force Geophysics Laboratory at SPO. This research was supported in part by the National Aeronautics and Space Administration through grants NSG-7511 and NAGW-91 by the Air Force Geophysics Laboratory through contract F19628-77-C-0104 to the University of Colorado.

\section{References}

Altrock, R. C. and Canfield, R. C.: 1974, Astrophys. J. 194, 733.

Altrock, R. C., November, L. J., Simon, G. W., Milkey, R. W., and Worden, S. P.: 1975, Solar Phys. 43, 33.

Berthomieu, G., Cooper, A. J., Gough, D. O., Osaki, Y., Provost, J., and Rocca, A.: 1980, in H. A. Hill and W. A. Dziembowski (eds.), Nonradial and Nonlinear Stellar Pulsation, Lecture Notes in Physics, Vol. 125, Springer, Berlin, p. 307.

Brandt, P. N.: 1969, Solar Phys. 7, 187.

Bumba, V.: 1970, Solar Phys. 14, 80.

Bumba, V. and Howard, R.: 1969, Solar Phys. 7, 28.

Deubner, F. L., Ulrich, R. K., and Rhodes, E. J., Jr.: 1979, Astron. Astrophys. 72, 177.

Gilman, P. A.: 1980, Highlights Astron. 5, 91.

Gough, D. O.: 1978, in G. Belvedere and L. Paterno (eds.), Workshop on Solar Rotation, Astrophys. Obsv. Catania, Palermo, p. 255.

Gough, D. O. and Toomre, J.: 1983, Solar Phys. 82, 401 (this volume).

Latour, J., Toomre, J., and Zahn, J.-P.: 1981, Astrophys. J. 248, 1081.

Latour, J., Toomre, J., and Zahn, J.-P.: 1983, Solar Phys. 82, 387 (this volume).

Lubow, S. H., Rhodes, E. J., Jr., and Ulrich, R. K.: 1980, in H. A. Hill and W. A. Dziembowski (eds.), Nonradial and Nonlinear Stellar Pulsation, Lecture Notes in Physics, Vol. 125, Springer, Berlin, p. 300.

McIntosh, P. S.: 1980, in M. Dryer and E. Tandberg-Hanssen (eds.), Solar and Interplanetary Dynamics, D. Reidel Publ. Co., Dordrecht, Holland, p. 25. 
November, L. J., Toomre, J., Gebbie, K. B., and Simon, G. W.: 1979, Astrophys. J. 227, 600.

November, L. J., Toomre, J., Gebbie, K. B., and Simon, G. W.: 1982, Astrophys. J. 258, 846.

Rhodes, E. J., Jr., Ulrich, R. K., Harvey, J. W., and Duvall, T. L.: 1981, in R. B. Dunn (ed.), Solar Instrumentation: What's Next?, Sacramento Peak Obs., Sunspot, p. 37.

Saastamoinen, J.: 1979, in E. Tengstrom and G. Teleki (eds.), 'Refractional Influences in Astrometry and Geodesy', IAU Symp. 89, 73.

Simon, G. W.: 1966, Astron. J. 71, 190.

Tarbell, T. D.: 1981, private communication.

Tarbell, T. D. and Smithson, R. C.: 1981, in R. B. Dunn (ed.), Solar Instrumentation: What's Next?, Sacramento Peak Obs., Sunspot, p. 491.

Toomre, J.: 1980, Highlights Astron. 5, 571. 